\title{
Simple and Efficient Furfural Production from Xylose in Media Containing 1-Butyl-3-Methylimidazolium Hydrogen Sulfate
}

\author{
Susana Peleteiro, ${ }^{\dagger}$ Andre M. da Costa Lopes, ${ }^{\ddagger}, \S$ Gil Garrote, ${ }^{\dagger}$ Juan Carlos Parajó, ${ }^{\dagger}$ \\ and Rafał Bogel-Łukasik**
${ }^{\dagger}$ Chemical Engineering Department, Faculty of Science, University of Vigo (Campus Ourense), Polytechnical Building, As Lagoas, 32004 Ourense, Spain
‡Unidade de Bioenergia, Laboratório Nacional de Energia e Geologia, 1649-038 Lisbon, Portugal
${ }^{\S}$ LAQV/REQUIMTE, Departamento de Química, Faculdade de Ciências e Tecnologia, Universidade Nova de Lisboa, $2829-516$ Caparica, Portugal

\begin{abstract}
The acidic 1-butyl-3-methylimidazolium hydrogen sulfate ([bmim $]\left[\mathrm{HSO}_{4}\right]$ ) ionic liquid was explored as both a reaction medium and a catalyst in the furfural production from xylose. Preliminary experiments were carried out at $100-140{ }^{\circ} \mathrm{C}$ for 15-480 min in systems containing just xylose dissolved in $[\mathrm{bmim}]\left[\mathrm{HSO}_{4}\right]$ in the absence of externally added catalysts. More than $95 \%$ xylose conversion was achieved when operating at 120 or $140{ }^{\circ} \mathrm{C}$ for 300 and 90 min, respectively; but just $36.7 \%$ of the initial xylose was converted to furfural. Operation in biphasic reaction systems (in the presence of toluene, methyl-isobutyl ketone or dioxane as extraction solvents) at $140{ }^{\circ} \mathrm{C}$ under selected conditions resulted in improved furfural production (73.8\%, $80.3 \%$, and $82.2 \%$ xylose conversion to furfural for the cited extraction solvents, respectively).
\end{abstract}

\section{INTRODUCTION}

The fast exhaustion of fossil resources and the need of safe supply of raw materials are major challenges to be addressed in the very near future. In this context, the development of environmentally and economically viable processes for manufacturing chemicals, fuels, and materials from widespread, renewable resources (among which lignocellulose plays a key role) is mandatory.

The biorefinery concept enables an efficient utilization of lignocellulosic materials via the fractionation of their polymeric components (cellulose, hemicellulose, and lignin), which are separated (intact or as derived products) to take advantage of the different chemical and physical properties of the resulting fractions.

Hemicellulosic polymers are composed of many sugar structural units. Among them, the most abundant is xylan, which is composed of $(1-4)$-linked $\beta$-D-xylopyranosyl residues. Typical lignocellulosic materials containing xylan are corncobs, hulls, straws, grasses, hardwoods, or some industrial byproducts (for example, bagasse or brewery spent grains). The acidic processing of xylan-containing substrates under mild conditions leads to a solid (mainly composed of cellulose and lignin) suitable for further processing, and to a liquid phase containing xylose. ${ }^{1}$ Dehydration of xylose leads to the formation of furfural $\left(\mathrm{OC}_{4} \mathrm{H}_{3} \mathrm{CHO}\right)$, which is a heterocyclic aldehyde that has been identified as a possible target product for biorefineries. ${ }^{2,3}$ Furfural is used for multiple purposes (for example, as a selective extraction agent, a solvent, an agrochemical, a flavoring agent, an intermediate in the manufacture of resins, and as the starting compound for the production of a broad range of chemicals). The manufacture of furfuryl alcohol accounts for most of the furfural demand, whereas other alcohols and furan derivatives are also commercialized. Furfural, which can also be converted to green fuels and coupling products, was selected as one of the value-added chemicals from biomass. ${ }^{4,5}$

The generation of furfural from xylose goes through a complex mechanism (Figure 1). In aqueous media, xylose can either undergo retroaldol fragmentation into acids, aldehydes, and ketones, or converted to intermediates whose chemical nature is controversial. ${ }^{6-8}$ In further reactions, furfural is first produced and then consumed ${ }^{9}$ by direct decomposition and/or by secondary reactions with itself, with xylose ${ }^{10}$ or with intermediates, leading to the formation of humins. ${ }^{11,12}$ The furfural yield is also decreased by increased xylose concentrations, because of the enhanced rate of side reactions.

Currently, the commercial production of furfural (performed in acidic, aqueous media) suffers from low yields and technological challenges related to corrosion, neutralization, and disposal of sludges. ${ }^{14}$ Several alternatives have been proposed to improve the production of furfural in aqueous media. In order to limit furfural decomposition, it can be removed from the reaction medium during the reaction, for example, by extraction.

Ionic liquids (ILs) are considered as an example of moresustainable solvents, which contribute to a greener processing of biomass, ${ }^{15-24}$ and are suitable for furfural production. Most studies performed on furan production from carbohydrates (including monosaccharides, oligosaccharides, or polysaccharides) in ILs (frequently, imidazolium salts) have been performed in the presence of catalysts such as Lewis acids, Brönsted acids, and solid acids. ${ }^{16,25}$ Generally, either taskspecific ILs with metals incorporated in the IL structure or

Received: May 13, 2015

Revised: August 10, 2015

Accepted: August 12, 2015

Published: August 12, 2015 